Article

\title{
Developing Professional Communication: The Construction of a Multimodal Understanding of Job Interviews
}

\author{
Wing Yee Jenifer Ho $\mathbb{D}$ \\ Department of English, City University of Hong Kong, Hong Kong, China; jenifer.ho@cityu.edu.hk
}

Received: 7 December 2018; Accepted: 13 January 2019; Published: 17 January 2019

\begin{abstract}
This article explores how online videos with a pedagogical focus can possibly make an impact on our current language teaching and learning practices. The affordance of videos to create multimodal content that can be shared with the public allows content creators to use a wide range of resources, such as spoken and written language, gestures, screen layout, etc., to create learning environments that can promote an awareness of a multimodal perspective to the understanding of a particular kind of professional communication context, such as job interviews, as illustrated in this article. By analyzing a series of videos on job interviews using multimodal semiotic analysis, I argue that these videos, which I call pedagogical vlogs, are helpful not only in terms of teaching the language skills required for job interviews, but also to help create a multimodal understanding of job interviews through the strategic orchestration of multiple semiotic modes. The popularity of pedagogical vlogs, as well as their affordance to provide lesson content created by the public, offer new possibilities for language teaching and learning, but it has yet only received scant attention from applied linguistics and language education researchers. This article aims to start a dialog on the pedagogical implications of this new form of learning so as to uncover the potentials offered by pedagogical vlogs in education.
\end{abstract}

Keywords: material design; mobile learning; multimodal design; multimodal semiotic analysis; vlogs

\section{Language Learning and Mobility}

The use of digital technologies in language learning is becoming more and more common. It has transformed how languages are taught and learnt in various formal and informal settings. People are no longer confined to a designated space and time in which to learn a new language. Digital technologies enable learners with diverse linguistic, cultural, and socioeconomic backgrounds to learn a language whenever, and wherever they like. Language learning no longer has to happen in a classroom; anywhere, and any kind of activities, can be sites for language learning. The blurring of the distinction between everyday life and language learning, or as Pachler et al. (2010) observe, the 'penetration' of mobile technologies into people's everyday lives, is a key feature of the modern language learning landscape.

In this article, I am concerned with the use of online videos as a resource for developing multimodal skills for communicating in professional contexts, one such context is job interviews. Attending a job interview in a foreign language is a challenge. Not only do students have to master the language required for the interview, but they also have to act in a way that is appropriate, in other words, to develop a multimodal understanding of interviews. A great deal of embodied performance is involved. Therefore, a multimodal approach to communication is needed to understand how a multimodal understanding of job interviews is constructed. This article is based on the understanding that all communications, learning included, are multimodal, meaning that different semiotic resources, 
including language, are deployed and orchestrated by the interlocutors to make meaning (Kress 2010). The research question that this article seeks to address is: What resources are mobilized in the focal language learning videos in order to facilitate multimodal learning of job interview language and skills?

The ubiquitous nature of video-sharing platforms such as YouTube has been a driving force of transforming the language classroom. Whilst online video-hosting sites such as YouTube have conventionally been regarded as a platform that provides entertainment in the form of music videos and funny video clips made by the general public, as digital content production is becoming more diverse, online video-hosting sites have now become our 'go-to' site if we want to learn how something is done, be it applying make-up, fixing a bicycle, or even getting you a job.

This article focuses on a series of five YouTube videos which aim to teach job interview skills in English. I begin the article by reviewing existing studies of the use of online videos in learning contexts, and then moving on to the use of multimodal social semiotics to analyze selected excerpts of one video with an aim to identify how linguistic and semiotic resources are used by the vlogger to create a kind of multimodal understanding of job interviews, not only on the language of asking and answering questions, but also on the multimodal aspects of it, such as gestures, attire, and setting related to a job interview.

\section{Online Videos as Resources for Learning}

Learning via watching online videos has conventionally been considered as a kind of out-of-class or informal learning practice (Benson 2015). It is sometimes seen as 'learning in the wild' (Conklin 2010) as learners are free to make learning decisions without the supervision of a teacher. In particular, the genre of video blogs (vlogs) is playing an increasingly influential role in the new learning landscape. Vlogs with a purpose of teaching, which I call 'pedagogical vlogs', warrant more research. The practice of vlogging has been around for about a decade, and the purpose of vlogging is diverse. In general, vlogs can be considered simply as the video version of text-based blogging on the Internet (Moor et al. 2010). However, as in the case of pedagogical vlogs, it is perhaps more helpful to see vlogging not as a single activity, but as a repertoire of genres and activities. The word 'vlogging' should be seen as "an umbrella term that covers a wide number of genres, including everything from short video footage of spontaneous, real-life, personal moments, to scripted and preplanned "shows" with characters, narratives, and professional acting" (Lange 2007). This definition of vlogging is one that is preferred in this article as it highlights the multifaceted and fluid nature of this activity, in which pedagogical vlogs is just one subgenre of vlogs. One feature of pedagogical vlogs which differs from other types of pedagogical videos is that they are created by one or two dedicated vloggers who post video lessons online regularly, and that there are a lot of interactions between the vlogger(s) and audience through the comments section of the video-hosting sites. Despite the variations between different types of vlogs, as discussed in Jones and Hafner (2012), vlogging brings about shifts in roles for the lay public to actively curate content which has the potential to reach a large audience. This affordance of vlogging warrants more research as it has the ability to influence how teaching and learning could be done in the future.

Even within the subgenre of pedagogical vlogs, there are wide variations. Some resemble the popular vlog genre in which the vlogger engages the audience in a monologue that is fast-paced, very much similar to a 'talk-show' where the vlogger uses semiotic resources, including language, to close the distance with the audience so that it is like talking to an intimate friend. On the other hand, some pedagogical vlogs are more pedagogically oriented, in which the vloggers try to re-enact classroom teaching through the medium of video. The series of pedagogical vlog featured in this article falls into the latter category in which the vlogger positions himself or herself as a teacher through various semiotic resources, details of which are discussed in the later section of this article. 


\section{The Design of Multimodal Learning Environments}

Although pedagogical vlogs are hosted on popular video-sharing platforms, vloggers take on the role of 'designers' of the learning environment. 'Design', in a multimodal social semiotics sense, refers to "the situated process in which a signmaker chooses semiotic resources and possible arrangements for semiotic entities to be produced to meet particular social functions or purposes" (Jewitt et al. 2016, p. 73). In the case of creating pedagogical vlogs, vloggers have to analyze the affordances and constraints of delivering lesson content using videos, and make decisions on how to maximize learning opportunities within the constraints of the video medium by carrying out an assessment of what semiotic resources should be used, when, and how. Using the explanation offered by Jones and Hafner (2012), affordances refer to what tools enable us to do, for instance, in the case of videos, to present information in a multimodal way and to disseminate the content to a wide range of audience in a short period of time. On the other hand, constraints refer to what tools prevent us from doing, and in the case of videos, one constraint could be the lack of face-to-face interaction between vloggers and audience. Vloggers, or in this case designers of learning environment, make meaning linguistically and semiotically, orchestrating the various resources at their disposal. The selection of semiotic resources to create pedagogical vlogs plays an important part in the design of the multimodal learning environment. As designers of learning environment, not only do vloggers need to gain an understanding of the affordances and constraints of the platform, they also have to strategically orchestrate the multimodal resources that are available so that the modal intensity of different resources, in different parts of the lessons, can realize the learning objectives in apt ways, by making certain aspects salient, and others less so. As observed by Norris (2004) when analyzing multimodal interactions, communicative modes take on different levels of intensity depending on the nature of the communication. For instance, in a telephone conversation, the intensity of spoken language is the strongest. We know this because it is the mode that the interlocutor at the other end of the telephone directly reacts to. Whilst this study does not deal with human-to-human interactions like Norris (2004) did, the methodological framework of multimodal interaction, such as the concept of modal intensity, does help us make sense of the learning environment featured in this study.

Previous research on the use of technologies for language teaching and learning has focused on issues such as usability (see, e.g., Stevenson and Liu 2010), effectiveness (see, e.g., Macaro et al. 2012), or how digital technologies have been changing learners' learning strategies (see, e.g., Qian et al. 2018). For instance, Stevenson and Liu (2010) focused on exploring the pedagogical usability of three online language learning sites, aimed at finding out "how learnable and usable the website is for learners" (p. 235); Macaro et al. (2012) offered a systematic review of 47 studies related to language teaching technologies and attempted to determine whether there are any direct benefits of using technologies for language education; Qian et al. (2018) identified emerging Chinese learning strategies which were made prominent with the use of mobile technologies. Nevertheless, only limited research focuses on the design of materials with a multimodal perspective which brings to the fore the fact that, in addition to language, there are other kinds of resources that content creators use to facilitate (language) learning. I argue that more attention has to be paid to the design of teaching materials, the pedagogical implications of the use of semiotic resources, and the way they are orchestrated to make meanings. Digital technologies such as videos and websites offer a wide range of semiotic resources for vloggers to utilize, leading to greater variation in the content, style, and aesthetics of sign-making practices (Adami 2018a). Vloggers can effectively use videos as a means to project a high level of self-identity by using a combination of semiotic resources, setting one vlogger apart from another. In other words, vloggers' voices can be constructed by their selection of apt resources. In the online world, especially in the marketplace of video-sharing platforms, having a unique voice is of crucial importance.

\section{Method}

To recap, the research question that this article seeks to address is: What resources are mobilized in the focal language learning videos in order to facilitate multimodal learning of job interview language 
and skills? By addressing this question, this article argues that pedagogical vlogs are helpful not only in terms of teaching the language skills required for job interviews, but also to help create a multimodal understanding of job interviews through the vloggers' strategic orchestration of multiple semiotic modes.

This article adopts the approach of multimodality which asserts that all communication involves the use of multiple modes, such as writing, speech, image, animations, gestures, etc., and that not one mode is superior or privileged over another. This understanding is particularly relevant in language teaching, as it was a common belief that linguistic modes (e.g., writing and speech) were the dominant modes, whereas other modes were peripheral. Multimodality challenges the belief of the superiority of language and recognizes the contributions of other modes as equally significant in a communicative context (Kress 2010). Of particular importance is the concept of meaning-making. When meaning is made, there has to be a 'maker', and hence agency is important in which the meaning-maker, or the sign-maker, has to make decisions to select apt modes to carry the meaning based on his/her interests, as well as the meaning potential of the resources available (Kress 2010). Adopting the approach of multimodality enables the analysis of the motivated choice of resources that the vlogger used in creating his or her videos, and how the orchestration of these resources creates a multimodal understanding of job interviews pedagogically.

This study is based on a series of videos on job interviews in English. Job interview was chosen as the professional context that I would like to focus on because it requires multiple skills for it to be successful, mastery of interview language being one such skill. Other skills such as manner, attitude, gestures, facial expressions, all play an important role to contribute to the overall success of an interview. It is also an important event that a lot of people would have to attend in the course of their lifetime, so it is a high-stake situation that warrants attention and research. Job interviews illustrate a kind of professional context whereby not only language skills are important, but the orchestration of semiotic modes also play a crucial role.

The study involved a qualitative approach to analyze five videos in the series. Multimodal semiotic analysis, informed by Social Semiotics, is used as an analytical framework. A social semiotic approach to multimodality is based on the notion of motivated sign (Kress 2010), which states that the relation between the signifier (e.g., the multimodal resources being mobilized) and signified (e.g., the intention to engage audience) is always motivated, recognizing the agency of the sign-maker, which is the vlogger in this case. As mentioned by Jewitt et al. (2016), the analytical starting point of multimodal semiotic analysis often involves a general description of the artefact, such as its context of use and general structure. The use of modes is then described and examined in detail, which leads to the issue of how the artefact concerned is designed.

This series of video is chosen for analysis because it focuses not only on the language skills required to excel in job interviews, but the video series also pays a great deal of attention to other resources such as gestures, which contribute to a multimodal understanding of the job interview genre, showing interview as an embodied performance. The videos were repeatedly viewed, and preliminary notes were made about the kinds of resources that were used in different parts of the video. After preliminary analysis, the videos were then divided into the macrostructure, as shown in Table 2 in Section 5.1, and the sections identified were treated as basic unit of analysis. In this article, the parts most relevant to the learning objectives of the video were analyzed in greater detail using multimodal semiotic analysis, while the parts less relevant, such as the vlogger's appeal to comments and signing up to the mailing list, were not analyzed.

\section{Ethical Issues}

Researching in online settings presents a unique set of ethical issues that are different from researching in face-to-face contexts. This research abides by the guidelines suggested by various professional bodies in educational research: 
Seeking consent would not normally be expected for data that have been produced expressly for public use. There is no consensus, however, as to whether those in online communities perceive their data to be either public or private, even when copyrights are waived. (British Educational Research Association (BERA) (2018) Ethical Guidelines for Educational Research, p. 10.)

Referring to the American Educational Research Association (AERA) (2011) Code of Ethics (2011), it is mentioned that:

Education researchers may conduct research in public places or use publicly available information about individuals (e.g., naturalistic observations in public places, analysis of public records, or archival research) without obtaining consent (American Educational Research Association (AERA) (2011) Code of Ethics, p. 151)

A useful guide is also provided by the Central University Research Ethics Committee (CUREC) Best Practice Guidance at the University of Oxford. When deciding whether informed consent is required, two criteria are important: (1) whether it is publicly available data (i.e., whether registration is required), and (2) whether the researched is a lay public or public figure (can be decided on a case-by-case basis). For the first criterion, the videos featured in this study were uploaded to YouTube in 2016, which are publicly available. For the second criterion, the vlogger concerned has a following of 1,720,974 people, and the focal video focused in this study has been viewed 1,279,241 times as at the time of writing, and, therefore, it can be argued that she is not a lay public, and her videos are meant to be watched by the public.

It can be seen that whether the focal videos belong to the public or private domain is not a clear-cut issue, so as the requirement of informed consent. At the preliminary observation stage, I checked the official website of the vlogger. She explicitly gives permission for anyone to use her videos as long as it is for a free project, and as long as the YouTube links of her videos are acknowledged. As an act of prudence, I sought to obtain informed consent from the vlogger, but there had been no reply. Therefore, given the guidelines and codes above, the statement from the vlogger's website, as well as the nonsensitive nature of this research, the data obtained is deemed appropriate for research purposes. Since the data is used for academic publications, according to the advice of University of Oxford Central University Research Ethics Committee (CUREC) (2018) at the University of Oxford, I decided to de-identify the vlogger in the analysis. This decision was made also after consulting the work of Codreanu and Combe (2018), who encountered a similar situation. Nevertheless, it has to be emphasized that the content of the video is already made available in the public domain, before this study even started; therefore, I did not disguise the vlogger's name as she already has a presence on YouTube. Readers may consult the links provided in the References section, as well as the Acknowledgement section to view the videos in question.

\section{Multimodal Semiotic Analysis of a Minilesson}

This section presents a multimodal semiotic analysis of five minilessons, all on the topic of job interviews. The vlogger that created the series of lessons on teaching English job interview skills is called Rachel. She is based in the United States. Rachel creates a wide variety of English teaching videos. The series of videos featured in this study is only one type of video that she creates, which features the genre of role play. Through browsing her YouTube channel, called Rachel's English (2016) I found that she uses a wide repertoire of techniques in creating minilessons, including role play, presentation, self-broadcasting, etc. The focal videos on job interviews is a five-part series entitled How to Interview for a Job in American English. It has been posted online since January 2016 and has accumulated over 12,080,000 views at the time of writing. This is another affordance of creating lesson content and distributing it by YouTube: the ability to reach millions of people in a short period of time. 


\subsection{Overview of the Videos}

The video series How to Interview for a Job in American English comprises of 5 videos. The length of videos $1-4$ is approximately $7 \mathrm{~min}$ long, with the exception of video 5 , which lasts for $16 \mathrm{~min}$. Table 1 shows the foci of each video:

Table 1. The foci of each video in the How to Interview for a Job in American English series.

\begin{tabular}{lll}
\hline Video 1 & $7: 04$ & Small talk, self-introduction (Tell me something about yourself) \\
\hline Video 2 & $7: 31$ & $\begin{array}{l}\text { Can you tell me how you heard about this position? What attracted you to our company? } \\
\text { What would you say is your greatest strength? }\end{array}$ \\
\hline Video 3 & $6: 40$ & When have you suffered a setback? What is your greatest weakness? \\
\hline Video 4 & $6: 43$ & Where do you see yourself in 5 years? Do you have any questions for me? \\
\hline Video 5 & $16: 16$ & Body language, humour, expression \\
\hline
\end{tabular}

The analysis of this video series focuses on the way different modal resources are mobilized strategically by Rachel in order to unpack how a multimodal understanding of job interviews is constructed and re-enacted in a role-play scenario. After preliminary examination of the series of five videos, the following macrostructure is identified (Table 2):

Table 2. The structure of a minilesson.

\begin{tabular}{l}
\hline A. Introduction to the video series \\
- Outlines the significance and difficulties of attending a job interview \\
\hline B. Logo of Rachel's English \\
\hline C. Introduction specific to the video \\
- $\quad$ Outlines the stressful nature of attending a job interview in a second language \\
\hline D. Role play of a part of a job interview \\
- $\quad$ Shows a change of setting and characters, attire, attitude, etc. \\
\hline E. Explanation \\
- $\quad$ Explains the purpose of a particular question in a job interview \\
- $\quad$ Explains the importance of a particular question in a job interview \\
- $\quad$ Provides on hample questions and responses \\
\hline Advises on how interviewees can practice answering that question on their own \\
\hline F. Conclusion (of the explanation) \\
Back to Role play (The role play-explanation-conclusion cycle continues for $2-3$ times in a video) \\
\hline G. Preview of the next video \\
\hline H. Concluding remarks \\
\hline I. Appeal to comments \\
\hline J. Appeal to signing up for her mailing list \\
\hline K. Thank the audience \\
\hline L. Advertisement for subscribing to premium courses \\
\hline
\end{tabular}

The macrostructure presented in Table 2 does not intend to provide a general understanding of the genre of pedagogical vlogs. As mentioned in Section 2, even within the genre of pedagogical vlogs, variations exist, and it is therefore not advisable to generalize the analysis to claim that it represents the structure of pedagogical vlogs. However, identifying the structure of the pedagogical vlog in question can help us contextualize the analysis that follows. 


\subsection{Mobilization of Linguistic and Semiotic Resources}

The following analysis, as shown in Table 3 , is based on the resources employed in different stages of one minilesson, video 1 , with a focus on sections A-F, as they are most relevant to the learning objectives of the videos. This analysis is solely based on part 1 of the five-part series, as all the other videos adopt a similar structure, with the exception of video 5.

Table 3. A multimodal view of the lesson.

\begin{tabular}{cccc}
\hline Time & Screen capture & $\begin{array}{l}\text { Narration by Rachel (other semiotic } \\
\text { modes are indicated by italics) }\end{array}$ & Explanatory notes \\
\hline interviews & $\begin{array}{l}\text { Today's video is part one in a series } \\
\text { that's all about preparing for a job } \\
\text { interview. }\end{array}$ & $\begin{array}{l}\text { Introduction (A) frames the video. } \\
\text { An upper-body shot of Rachel to } \\
\text { the left of the screen, title is shown } \\
\text { on the right in white that contrasts } \\
\text { sharply with the black } \\
\text { background }\end{array}$ \\
\hline
\end{tabular}

\begin{tabular}{|c|c|c|c|}
\hline 0:19 & $\begin{array}{l}\text { Rachel's English } \\
\text { American English Pronunciation Guide }\end{array}$ & & $\begin{array}{l}\text { The logo (B) is shown being } \\
\text { coloured in pencil before turning } \\
\text { into a coloured logo, with the } \\
\text { sound of whistles in the } \\
\text { background }\end{array}$ \\
\hline & \multicolumn{3}{|l|}{ How to Interview for a Job in American English, part 1/5 } \\
\hline $0: 53$ & How to Interview for a Job in American English, part 1/5 & $\begin{array}{l}\text { Interviewing for a new job can be a } \\
\text { huge source of stress and anxiety. } \\
\text { And if you're interviewing for a job in } \\
\text { a non-native language, the stress can } \\
\text { be even higher. In this video, you'll } \\
\text { see me interview for a job. } \\
\text { Throughout the interview we'll } \\
\text { discuss some of the most common } \\
\text { interview questions and how to } \\
\text { answer them. You'll also learn some } \\
\text { basic information to get you started } \\
\text { on creating your own answers to } \\
\text { these questions. Let's begin. }\end{array}$ & $\begin{array}{l}\text { Introduction (C) shows Rachel } \\
\text { engaging with the audience by } \\
\text { looking directly at them and using } \\
\text { the second-person pronoun 'you' } \\
\text { to address the audience. }\end{array}$ \\
\hline 1:02 & & & $\begin{array}{l}\text { Role play (D) is not indicated } \\
\text { linguistically. It is indicated by a } \\
\text { variety of semiotic resources, e.g., } \\
\text { the formal suits worn by the } \\
\text { characters, the office setting with a } \\
\text { computer, pen, and paper. }\end{array}$ \\
\hline & r a Job in American English, part $1 / 5$ & & \\
\hline
\end{tabular}


Table 3. Cont.

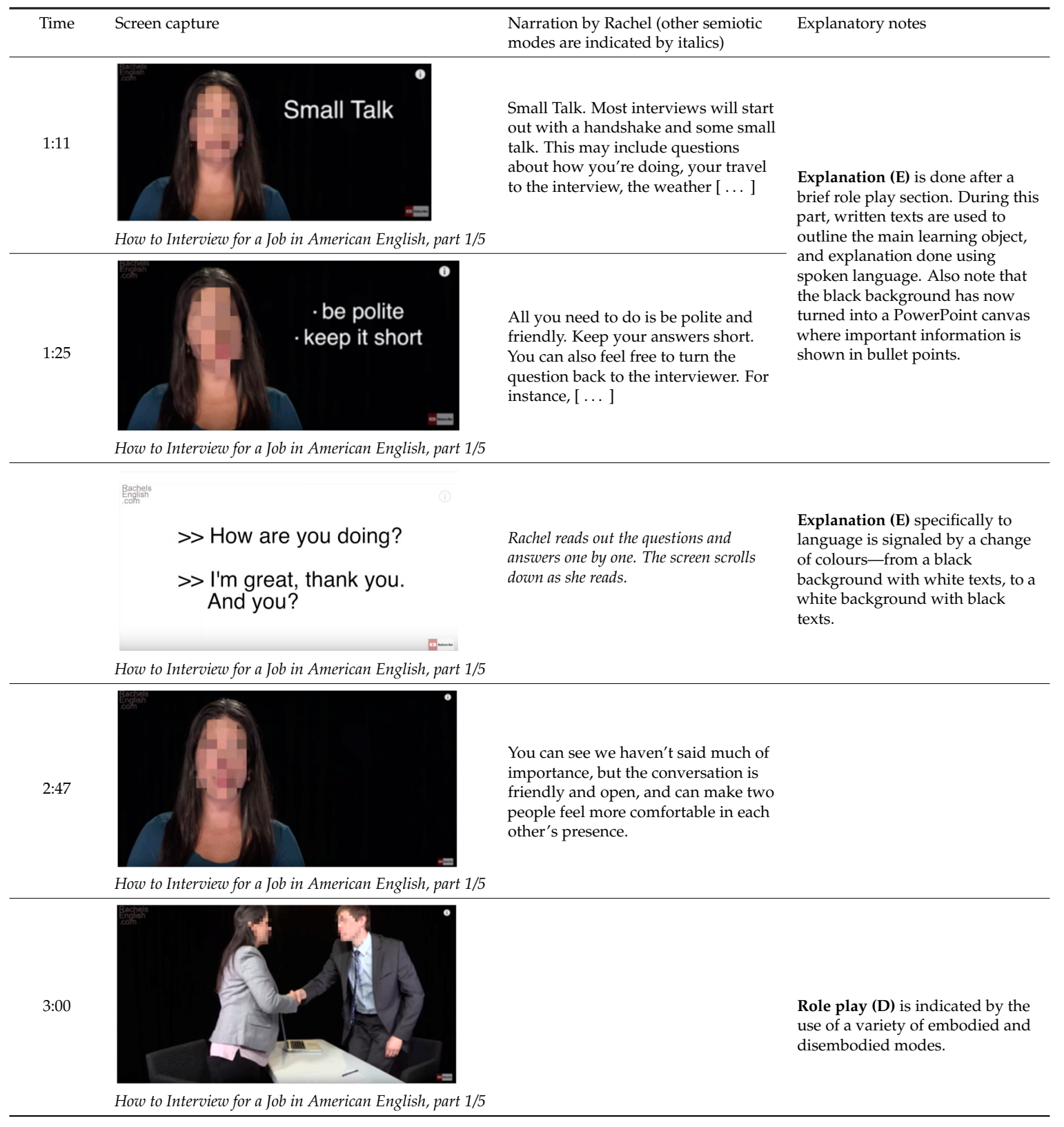

The learning objectives of the video, as mentioned by Rachel, were to: (1) discuss some of the most common interview questions; (2) learn how to answer them; (3) learn how to create answers to these questions. These are seemingly linguistically oriented questions. In bookstores all around the world, it is not difficult to find books covering similar language points specifically on job interviews. Here, the concept of modal affordance would be a helpful way to understand how learning job interview questions in books differs from learning them through videos. Modal affordance, as explained by Jewitt et al. (2016), refers to the fact that different modes have different potentials for making meaning due to their differences in material and social histories, of how they have been used in a specific context. The affordances offered by written language differ from what spoken language can offer, and in turn images can offer a different set of affordances for meaning-making. If we examine the affordances of books and videos, we will find that although these two media seemingly cover a similar kind of content, in this case, the learning of job interview questions, the affordances of videos allow the multimodal aspect of job interviews to be shown by means of role play. As discussed in Jones and Hafner (2012), videos differ from other media as they are comprised of sequence of images, which are 
capable of presenting visual information using the logic of the screen, as well as textual information using the logic of speech and writing. Furthermore, videos have pace which can determine how quickly or slowly information is presented, an affordance not seen in print media. Not only can viewers of the video learn how to ask and answer questions in an interview using spoken language, through immersing themselves in the video series, they are also able to learn how to present themselves in a job interview, such as the way of speaking, gestures, attire, and be familiarized with a typical job interview flow and settings, contributing to a multimodal understanding of job interviews; whereas in books, only texts and images can be used to depict the multimodal nature of job interviews, other resources such as the way of speaking and gestures-in-action are lost due to the different affordances that a print medium offers.

Meanings can be made by using both embodied and disembodied modes (Norris 2004). As can be seen from the analysis above, Rachel uses a variety of embodied modes, such as gesture, eye contact, facial expressions, as well as disembodied modes, such as the setting of the scene, placement of objects, etc., to frame different parts of the minilesson. For instance, snippets of role play are inserted at different points throughout the minilesson. Instead of using linguistic means, such as written texts or spoken language, to indicate that this is a role play explicitly, semiotic resources are used instead to frame this part of the lesson as a role play, as an enactment of a job interview. When the role play is over, the explanation is signaled by Rachel standing on the left-hand side of the screen, indicating that she will now unpack what was shown in the role play and explain some of the important learning points. The modal intensity alternates between these embodied and disembodied modes in a strategic way so that the flow of the lesson is not disrupted unnecessarily, i.e., by stopping the lesson and stating 'this is a role play', or 'this is an explanation of language points', the flow of the lesson would be disrupted, as this kind of framing can be done by using different modal resources to make the learning objective salient, which in turn directs learners' attention in a certain way. This alternation helps create a learning environment that is engaging and authentic, providing a first-person experience of attending a job interview, not as an observer.

Engagement of audience is promoted by the use of embodied modes. In the explanation section, Rachel explains how to tackle common interview questions by using second-person pronouns 'you' and 'we' in order to engage audience by directly talking to them. She looks at the camera, as if she was looking at the audience in the eye as she explains. This is a kind of demand image which demands attention from viewers (Kress and Van Leeuwen 2006; Jones and Hafner 2012). Furthermore, the distance of the shot, which in this case is a medium shot, signifies a certain degree of personal distance, but close enough to be in our personal space to demand attention. The whole video is shot mostly at eye level, which conveys an egalitarian power relation between Rachel and the learners. Nevertheless, Rachel also uses linguistic means to position herself as a 'teacher', by giving advice using short and concise imperatives that use a firm tone, such as:

All you need to do is be polite and friendly. Keep your answers short. You can also feel free to turn the question back to the interviewer.

If you're preparing for an interview, practice talking about yourself and your work history. Record yourself with a video camera if possible. Make sure it's not too long, maybe around a minute. Go back and listen or watch, and write down phrases that worked well to use again.

By using these linguistic devices, together with the tone, pitch, and speed of her voice, she positions herself as an authoritative voice that learners can trust, but at the same time, a teacher that is 'down-to-earth' by showing willingness to engage and involve the audience.

\section{Implications to Language Teaching and Learning}

From the above analysis, it can be seen that the design of a multimodal learning environment requires semiotic work from Rachel. Semiotic work is a term to describe the sign-maker's agentive, purposeful actions which lead to the creation of new meanings for the sign-maker and the resources 
that he/she uses (Kress 2016; personal communication). From this understanding, it can be seen that Rachel utilized her full repertoires of multimodal resources, which included embodied modes as well as disembodied modes, to create her minilessons. The affordances of video to show a 'job interview in action' illustrates how different resources are orchestrated in an interview, which in turn facilitated a multimodal understanding of job interviews using English as a medium of communication. The affordances of videos to present visual and textual information using the spatial and simultaneous logic of the screen, as well as the linear and sequential logic of speech and writing have made it an effective medium to carry pedagogical content (see Jones and Hafner (2012) for a detailed discussion of the affordances of videos).

This study offers us some implications to language teaching and learning from the perspective of material design. The design of language teaching materials, in the past, was only possible by publishers. Now, the affordances and ubiquity of digital technologies allow the lay public to generate content on the web. This move from the 'read-only web' to the 'read-write web' (Jones and Hafner 2012) has generated exciting new possibilities which allow for wider access and participation in the field of language teaching and learning, resulting in greater variation and creativity in material design as a wider range of sign-making practices are used. Vloggers as teachers are able to, or are encouraged to, engage in a higher level of self-expression through exhibiting different styles and aesthetics in the design of their virtual 'schools' (see Adami (2018a) for a discussion of the role of digital platforms in shaping blog users' self-styling practices).

Recognizing the multimodal nature of learning has great implications for the development of self-directed language learning materials, as in the YouTube videos featured in this study. As mentioned, the field of language teaching and learning has seen an ideological preoccupation with language in the past, largely because of the limited availability of technology that impeded the use of modes in addition to language. However, the situation has now changed due to the ubiquity of technology that affords more effective use of modes other than language. This wider access to technology has influenced teaching and learning practices from a focus on language to other semiotic resources. If the goal of language learning is to be able to communicate with other people effectively, a multimodal approach to language learning is necessary. Block (2014) warned against the "lingual bias", which refers to "the tendency to conceive of communicative practices exclusively in terms of the linguistic (morphology, syntax, phonology, lexis)" (p. 56). Therefore, there is a need to rethink the current practices of teaching and learning that have been dominated by the use of linguistic modes. A sole focus on speech and writing would miss out on a lot of communication that happened using other semiotic modes, such as images, colours, the layout of the screen, etc. It is particularly evident in an era of mobility in which people are often in situations where they share few linguistic resources with other people (see Adami (2018b) research on communication in the market).

\section{Conclusions}

The use of digital tools such as YouTube videos to learn is becoming more common, thanks to the ubiquity of Web 2.0 technologies, which allows a variety of sign-making practices, such as different forms of language teaching videos, to be posted on the Internet for all to use. This emerging learning practice has the potential to dramatically change the way how knowledge is created and consumed, and so it warrants more research to understand the pedagogical implications it entails. This article has focused on how a vlogger uses her full repertoire of embodied and disembodied modes to create a learning environment which promotes a multimodal understanding of job interviews using English as a medium. Through an analysis of the structure and the modes used in the core part of a minilesson, it is seen that although the primary objectives of the video emphasized the linguistic aspect of job interviews, a multimodal understanding of interview, such as the use of appropriate gestures, way of speaking, attire, etc., are also shown in the lesson. The vlogger has also strategically used a variety of modes to frame different parts of the lesson so that the flow of the lesson is not disrupted 
unnecessarily, which is another way to promote an embodied, multimodal experience of job interviews to the audience.

This article argued that learning is a multimodal and embodied activity. Vloggers as content designers have to make decisions regarding the medium to carry lesson content. This decision is made based on an assessment of the affordances and constraints of the medium. Vloggers also have to select apt modal resources to convey meaning by assessing what a mode enables or prevents the vlogger to do, as well as to determine the modal intensity in different parts of the lesson so that the learning objectives are clearly foregrounded and met. Furthermore, vloggers have to 'design' the overall orchestration of embodied and disembodied modes to frame the various parts of the lesson, as well as to engage the audience.

Pedagogical vlogs represent a hybrid genre which may contain elements of teacher talk, acting, casual chat, live broadcast, etc. Not only is it an underexplored area in language education, the significance and implications of pedagogical vlogs cannot be underestimated. As digital technologies have the potential to allow learners to assume a higher level of agency, pedagogical vlogs can, potentially, contribute to what Little and Thorne (2017) called a 'rewilding' of language education, which involves knowledge production in the process of learning. This article only focused on one type of pedagogical vlogs which is more pedagogically oriented than other kinds of vlogs. To date, there is only little understanding of this complicated yet dynamic genre. Undoubtedly, pedagogical vlogs are playing an increasingly prominent role in language teaching and learning, and, therefore, more research is needed to understand, for instance, the multimodal features of these vlogs, the learning experience that they create, or their potentials to be used to supplement classroom language teaching in order to 'rewild' language education, by encouraging learners to discover learning resources relevant to them.

Funding: This research received no external funding.

Acknowledgments: The author would like to thank the reviewers for their supportive feedback, which can greatly improve the quality of this manuscript. Thanks must also be given to Rachel's English (https: / www.youtube. $\mathrm{com} /$ user/rachelsenglish) which provides the materials for this paper.

Conflicts of Interest: The author declares no conflicts of interest.

\section{References}

Adami, Elisabetta. 2018a. Styling the self online: Semiotic technologization in weblog publishing. Social Semiotics 28: 601-22. [CrossRef]

Adami, Elisabetta. 2018b. Multimodal sign-making in today's diversity: The case of Leeds Kirkgate Market. In Making Signs, Translanguaging Ethnographies: Exploring Urban, Rural and Educational Spaces. Edited by Sherris Ari and Elisabetta Adami. Bristol: Multilingual Matters, pp. 26-44.

American Educational Research Association (AERA). 2011. Code of Ethics. Available online: http:/ /www.aera. net/Portals/38/docs/About_AERA/CodeOfEthics(1).pdf (accessed on 1 November 2018).

Benson, Phil. 2015. Commenting to Learn: Evidence of Language and Intercultural Learning in Comments on YouTube Videos. Language Learning \& Technology 19: 88-105. Available online: http:/ /llt.msu.edu/issues/ october2015/benson.pdf (accessed on 1 December 2018).

Block, David. 2014. Moving beyond "lingualism": Multilingual embodiment and multimodality in SLA. In The Multilingual Turn: Implications for SLA, TESOL and Bilingual Education. Edited by Stephen May. New York and London: Routledge, pp. 54-77.

British Educational Research Association (BERA). 2018. Ethical Guidelines for Educational Research, 4th ed. Available online: https:/ / www.bera.ac.uk/wp-content/uploads/2018/06/BERA-Ethical-Guidelines-forEducational-Research_4thEdn_2018.pdf?noredirect=1 (accessed on 1 November 2018).

Codreanu, Tatiana, and Christelle Combe. 2018. Glocal Tensions: Exploring the dynamics of intercultural communication through a language learner's vlog. In Screens and Scenes: Multimodal Communication in Online Intercultural Encounters. Edited by Kern Richard and Christine Develotte. New York: Routledge, pp. 40-61.

Conklin, James. 2010. Learning in the wild. Action Learning: Research and Practice 7: 151-66. [CrossRef] 
Jewitt, Carey, Jeff Bezemer, and Kay O'Halloran. 2016. Introducing Multimodality. London; New York: Routledge. Jones, Rodney H., and Christoph A. Hafner. 2012. Understanding Digital Literacies: A Practical Introduction. London: Routledge.

Kress, Gunther. 2010. Multimodality: A Social Semiotic Approach to Contemporary Communication. London: Routledge. Kress, Gunther R., and Theo Van Leeuwen. 2006. Reading Images: The Grammar of Visual Design, 2 nd ed. London: Routledge.

Lange, Patricia G. 2007. The vulnerable video blogger: Promoting social change through intimacy. The Scholar and Feminist Online 5: 1-5.

Little, David, and Steven L. Thorne. 2017. From Learner Autonomy to Rewilding: A Discussion. In Learner Autonomy and Web 2.0. Edited by Marco Cappellini, Tim Lewis and Annick Rivens Mompean. Sheffield: Equinox Ebooks Publishing, pp. 12-35.

Macaro, Ernesto, Zöe Handley, and Catherine Walter. 2012. A systematic review of CALL in English as a second language: Focus on primary and secondary education. Language Teaching 45: 1-43. [CrossRef]

Moor, Peter J., Ard Heuvelman, and Ria Verleur. 2010. Flaming on YouTube. Computers in Human Behavior 26: 1536-46. [CrossRef]

Norris, Sigrid. 2004. Analyzing Multimodal Interaction: A Methodological Framework. London: Routledge.

Pachler, Norbert, Ben Bachmair, and John Cook. 2010. Mobile Learning: Structures, Agency, Practices. New York: Springer.

Qian, Kan, Nathaniel Owen, and Stephen Bax. 2018. Researching mobile-assisted Chinese-character learning strategies among adult distance learners. Innovation in Language Learning and Teaching 12: 56-71. [CrossRef]

Rachel's English. 2016. How to Interview for a Job in American English, Part 1-5. YouTube. Available online: https:/ / www.youtube.com/watch?v=yBtMwyQFXwA (accessed on 1 November 2018).

Stevenson, Megan P., and Min Liu. 2010. Learning a Language with Web 2.0: Exploring the use of social networking features of foreign language learning websites. CALICO Journal 27: 233-59. [CrossRef]

University of Oxford Central University Research Ethics Committee (CUREC). 2018. Internet-Based Research (IBR). Available online: https://researchsupport.admin.ox.ac.uk/sites/default/files/researchsupport/ documents / media/bpg_06_internet-based_research_v._5.2.pdf (accessed on 1 November 2018).

(C) 2019 by the author. Licensee MDPI, Basel, Switzerland. This article is an open access article distributed under the terms and conditions of the Creative Commons Attribution (CC BY) license (http://creativecommons.org/licenses/by/4.0/). 